\title{
Compression of Hyperspectral Images using Discerete Wavelet Transform and Tucker Decomposition
}

\author{
A. Karami ${ }^{\# 1}$, Student Member ,IEEE,M. Yazdi ${ }^{\# 1}$, Member, IEEE, and G. Mercier ${ }^{\# 2}$, Senior Member, \\ IEEE, \\ ${ }^{1}$ Department of Communications and Electronics, School of Electrical and Computer Engineering, \\ Shiraz University, Shiraz, Iran \\ ${ }^{2}$ Telecom Bretagne, Technopole Brest Iroise, CS 83818, 29238 Brest Cedex. \\ \{ akarami ,yazdi \}@shirazu.ac.ir, gregoire.mercier@telecom-bretagne.eu
}

\begin{abstract}
The compression of hyperspectral images (HSIs) has recently become a very attractive issue for remote sensing applications because of their volumetric data. In this paper, an efficient method for hyperspectral image compression is presented. The proposed algorithm, based on Discrete Wavelet Transform and Tucker Decomposition (DWT-TD), exploits both the spectral and the spatial information in the images. The core idea behind our proposed technique is to apply TD on the DWT coefficients of spectral bands of HSIs. We use DWT to effectively separate HSIs into different sub-images and TD to efficiently compact the energy of subimages. We evaluate the effect of the proposed method on real HSIs and also compare the results with the well-known compression methods. The obtained results show a better performance of the proposed method. Moreover, we show the impact of compression HSIs on the supervised classification and linear unmixing.
\end{abstract}

Index Terms- Hyperspectral Images, Tucker Decomposition, Wavelet Transform, Compression, Noise Reduction

\section{INTRODUCTION}

HSIs are used in different practical applications such as the detection of the earth's surface, soil type analysis, agriculture and forest monitoring, environmental studies and so on [1].

There are two types of redundancy in HSIs: spatial and spectral redundancies. However, the spectral correlation (band redundancy) is generally but not always stronger than spatial correlation [2]. Several compression methods have recently been proposed which can be classified into two main types: lossless and lossy compression methods. Lossless compression can only provide limited compression ratios and the maximum achievable order is around three times (3:1) [3]. This ratio is not a reasonable value in many practical applications especially in remote sensing.

Traditional compression algorithms for HSIs have been considered the spectral value in a feature space whose dimensions were spectral bands. Then, dimension reduction was often applied (by means of Principal Components Analysis (PCA) or Independent Component Analysis (ICA) [4]. However, they did not consider the spatial correlation when focusing on the spectral decorrelation. Therefore, 3D wavelet-based techniques such as Set Partitioning in Hierarchical Trees (SPIHT) algorithm and Set Partitioned Embedded bloCK (SPECK) for hyperspectral image compression have been proposed to exploit a joint consideration of the spatial and spectral correlations [5]. It has been shown in [5] that 3D-SPECK is better than 3DSPIHT to achieve an efficient compression. In [6], a PCA based method in conjunction with JPEG2000 for compressing HSIs was introduced. The results reveal that the performance of the method is superior to that of the spectral DWT and the best PCA performance occurs when a reduced number of PCs are retained and encoded. Another compression algorithm based on JPEG2000 for HSIs was proposed in [7]. The algorithm can be applied for lossy and near-lossless compression applications in one single tool. It was also shown that the proposed scheme has a negligible effect on the results of selected applications (e.g. hard classification, spectral unmixing, and anomaly detection). In [8] a new lossy-to-lossless coder based on 3D Tarp-based Coding with classification for Embedding (TCE) coupled with the reversible integer-valued Karhunen-Loève transform (KLT) was introduced. The proposed method closely matches the lossy performance of JPEG2000 and outperformed JPEG2000 at lossless compression. An HSIs 
compression method based on band-of interest BOIpreserving-based was proposed in [9]. Some bands of HSIs are more important in the specific applications, and BOI selection methods can be chosen according to application requirements. BOI and non-BOI bands are respectively compressed with low distortion and high distortion. In [10] the impact of lossy compression on spectral unmixing, and supervised classification using Support Vector Machine (SVM) was investigated. It was shown that for certain compression techniques, a higher compression ratio (CR) may lead to more accurate classification results.

Some compression methods currently consider HSIs as 3D data. Those compression methods are called a third-order tensor: two spatial dimensions and one spectral dimension. They try to take into account the spatial and spectral correlation of HSIs simultaneously and not alternatively as is the case for the above techniques [11]. Several tensor decompositions have been introduced in [12]. One of the most popular tensor decompositions is the Tucker decomposition (TD), which has been used for the compression of HSIs. TD allows selecting any values for each dimension of the core tensor, which will be defined as $\left(J_{1} \times J_{2} \times J_{3}\right)$ in the next section and helps to obtain a higher compression ratio. We propose a new HSIs compression algorithm based on discrete wavelet transform (DWT) and TD . Applying 2DWT to each spectral band will take care of first stage compression by using (9/7) biorthogonal wavelet. Next, TD is applied to the four wavelet subimages of the HSIs in order to achieve more CR. Finally, adaptive arithmetic coding (AAC) is used for coding the elements of the core tensors.

We compare the proposed method with the best known techniques, such as the 3D-SPECK algorithm [5], and both PCA and JPEG2000 [6]. Our experimental results over the two most used HSIs (AVIRIS datasets: Cuprite and Moffett Field) demonstrate and confirme the effectiveness of our algorithm in providing a much smaller MSE for the desired set of CRs, especially when the CR is selected higher than 160 , in this case the performance of the proposed algorithm is significantly better than the other techniques. The proposed method also increases the pixel-based supervised classification accuracy.

The rest of the paper is organized as follows. Section II briefly reviews the DWT and TD algorithms. Then, Section III introduces the proposed algorithm. The computational complexity of the proposed algorithm is provided in Section IV. Experimental results are shown in Section V to demonstrate the performance of the proposed compression algorithm, and the final conclusions are extracted in Section VI.

\section{3D REPRESENTATION OF HYPERSPECTRAL IMAGES}

HSIs are the images generated by the imaging spectrometer by collecting image data simultaneously in hundreds of spectral bands or frequencies ( e.g. 5-10 nm spectral width) that reach a nearly contiguous spectral record. The large amount of bands increases complexity and time of processing. HSIs can be represented as a third dimensional data $\underline{\mathbf{X}} \in \mathbf{R}^{I_{1} \times I_{2} \times I_{3}}$, as shown in Fig. 1 .

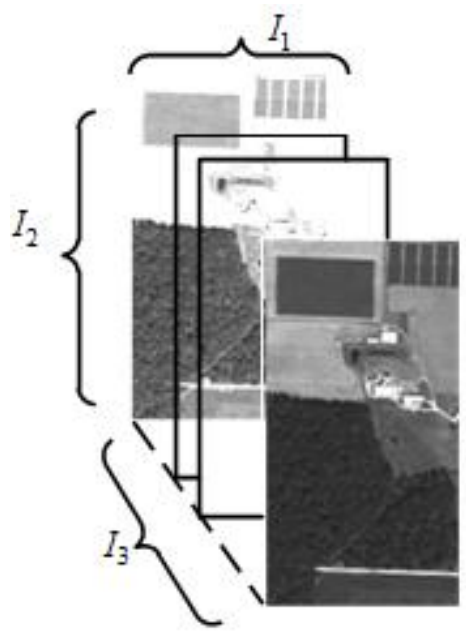

Fig. 1. Third-dimensional modeling of HSIs.

Unlike natural images, HSIs have two types of correlation simultaneously, which are the spatial correlation within images and the spectral correlation between spectral bands. The spectral correlation is generally stronger than spatial correlation. The average correlation coefficient $\bar{p}$ between two spectral bands of 3D-data cube can be measured as follows:

$$
\begin{aligned}
& \text { For } i_{3}=1 \text { to } I_{3}-1 \\
& \rho\left(i_{3}\right)=\frac{\operatorname{cov}\left(\underline{\mathbf{X}}\left(:,:, i_{3}\right), \underline{\mathbf{X}}\left(:,:, i_{3}+1\right)\right)}{\sqrt{\operatorname{var}\left(\underline{\mathbf{X}}\left(:,:, i_{3}\right)\right)} \sqrt{\operatorname{var}\left(\underline{\mathbf{X}}\left(:,,: i_{3}+1\right)\right)}} \\
& \text { End } \\
& \bar{p}=\frac{1}{I_{3}-1} \sum_{i_{3}=1}^{I_{3}-1} \rho\left(i_{3}\right)
\end{aligned}
$$

In most HSIs datasets, such as AVIRIS data cube, the value of $\bar{p}$ is higher than 0.90 which means that the spectral correlation among bands is very high.

Exploiting both of spectral and spatial correlations is the key for the success of a compression algorithm. In this paper a hybrid scheme 
based on DWT and TD for compression of HSIs is introduced. DWT and TD are briefly introduced in the next sections.

\section{A. Discrete wavelet transform (DWT)}

The DWT has successfully been used in many image processing applications including noise reduction, edge detection, and compression [13]. Indeed, the DWT is an efficient decomposition of signals into lower resolution and details. From the deterministic image processing point of view, DWT may be viewed as successive low-pass and high-pass filtering of the discrete time-domain signal. At each level, the high pass filter produces detailed information (horizontal (H), vertical (V) and diagonal (D) information), while the low pass filter associated with scaling function produces the approximate (A) information. We apply a two dimensional DWT to each band of HSIs. If each image band has $I_{1}$ rows and $I_{2}$ columns, then after applying the 2DWT, we obtain four sub-band images (A, H, V, D), each having $\frac{I_{1}}{2}$ rows and $\frac{I_{2}}{2}$ columns.

The A sub-band images have the highest energy among all the coefficients of the other sub-band images (see Fig.2).

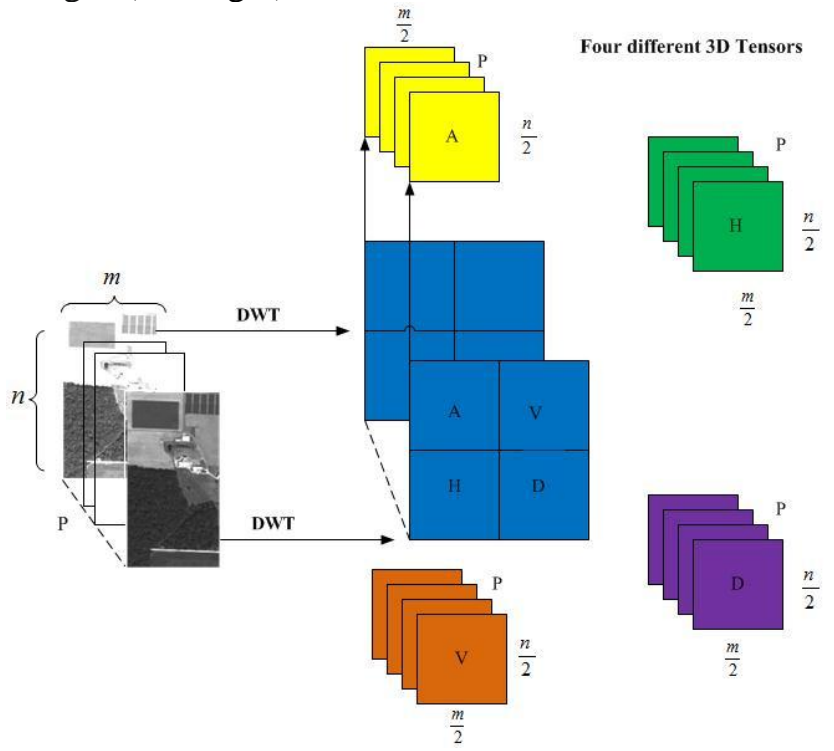

Fig. 2. New decomposition scheme in the proposed method for HSIs

The 2DWT of function $f(x, y)$ with of size $I_{1}$ and $I_{2}$ can be shown as:

$$
\begin{aligned}
& W_{\varphi}\left(i_{1}, i_{2}\right)=\frac{1}{\sqrt{I_{1} I_{2}}} \sum_{y=0}^{I_{2}-1} \sum_{x=0}^{I_{1}-1} f(x, y) \varphi_{i_{1}, i_{2}}(x, y) \\
& W_{\psi}{ }^{k}\left(j, i_{1}, i_{2}\right)=\frac{1}{\sqrt{I_{1} I_{2}}} \sum_{y=0}^{I_{2}-1} \sum_{x=0}^{I_{1}-1} f(x, y) \psi_{j, i_{1}, i_{2}}^{k}(x, y)
\end{aligned}
$$

$k=\{H, D, V\}$

$\varphi_{i_{1}, i_{2}}(x, y)=\varphi\left(x-i_{1}, y-i_{2}\right)$

$=\sum_{m} h_{\varphi}\left(m-2 i_{1}\right) \sqrt{2} \varphi(2 x-m) \times$

$\sum_{n} h_{\varphi}\left(n-2 i_{2}\right) \sqrt{2} \varphi(2 x-n)$

$\psi_{j, i_{1}, i_{2}}^{H}(x, y)=2^{\frac{j}{2}} \psi^{H}\left(2^{j} x-i_{1}, 2^{j} y-i_{2}\right)=$

$2^{\frac{j}{2}} \sum_{m} h_{\psi}\left(m-2 i_{1}\right) \sqrt{2} \psi\left(2^{j+1} x-m\right) \times$

$\sum_{n} h_{\psi}\left(n-2 i_{1}\right) \sqrt{2} \psi\left(2^{j+1} x-n\right)$

$\varphi$ is called scaling function. The $W_{\varphi}\left(i_{1}, i_{2}\right)$ coefficients define an approximation of $f(x, y)$. The $W_{\psi}\left(j, i_{1}, i_{2}\right)$ coefficient add horizontal, vertical and diagonal details, normally $i_{1}=i_{2}=2^{j}$ is selected and so that $j=0,1,2, \ldots, J-1[14]$. The $h_{\varphi}$ and $h_{\psi}$ are called wavelet filters. For the choice of wavelet filtering, the (9/7) biorthogonal wavelet which is used in JPEG2000, is selected in order to improve the compression ratio[15]. Filter coefficients of (9/7) biorthogonal wavelet are shown in Table $1[16,17]$.

Table 1 9/7 Biorthogonal Wavelet Filter

\begin{tabular}{|c|c|c|}
\hline$n$ & $h_{\varphi}[n]$ & $h_{\psi}[n]$ \\
\hline 0 & 0.7885 & 0.8527 \\
\hline \pm 1 & 0.4181 & 0.3774 \\
\hline \pm 2 & -0.04069 & -0.111 \\
\hline \pm 3 & -0.06454 & -0.02385 \\
\hline \pm 4 & & 0.03783 \\
\hline
\end{tabular}

The inverse 2DWT is formulated as follows:

$$
\begin{aligned}
& f(x, y)=\frac{1}{\sqrt{I_{1} I_{2}}} \sum_{i_{2}} \sum_{i_{1}} W_{\varphi}\left(i_{1}, i_{2}\right) \varphi_{i_{1}, i_{2}}(x, y)+ \\
& \frac{1}{\sqrt{I_{1} I_{2}}} \sum_{k=\{H, D, V\}} \sum_{j=0}^{J-1} \sum_{i_{2}} \sum_{i_{1}} W_{\psi}^{k}\left(i_{1}, i_{2}\right) \psi_{j, i_{1}, i_{2}}^{k}(x, y)
\end{aligned}
$$

In lossy compression, we can ignore the detailed sub-bands (for example $\mathrm{H}, \mathrm{V}$ and $\mathrm{D}$ ) or preserve only important detailed information. In order to achieve a higher compression ratio, we can still decompose the images in more than one level.

\section{B. Nonnegative Tucker Decompositions}

In this section a brief review of the Tucker model is presented. Important notations are shown in Table 2. 
Table 2 Notations

\begin{tabular}{|c|c|}
\hline Notation & Description \\
\hline $\mathbf{R}^{n}$ & $n$-dimensional real vector space \\
\hline$\underline{\mathbf{Y}}$ & third order tensor \\
\hline $\mathbf{Y}_{(n)}$ & $n$-mode matricization of tensor $\underline{\mathbf{Y}}$ \\
\hline $\mathbf{A}^{(n)}$ & $n$-mode matrix in Tucker model \\
\hline$\circ$ & outer product \\
\hline$\times_{n}$ & $n$-mode product of a tensor by matrix \\
\hline
\end{tabular}

The third- order TD tensor (see Fig.3) is described as a decomposition of a given third order tensor $\underline{\mathbf{Y}} \in \mathbf{R}^{I_{1} \times l_{2} \times l_{3}}$ into an unknown core tensor $\underline{\mathbf{G}} \in \mathbf{R}^{J_{1} \times J_{2} \times J_{3}}$ multiplied by a set of three unknown component matrices where, $\quad \mathbf{A}^{(n)}=\left[a_{1}^{(n)}, a_{2}^{(n)}, \ldots, a_{J_{n}}^{(n)}\right] \in \mathbf{R}^{I_{n} \times J_{n}}$ $(n=1,2,3)$ represent common factors [18].

$\underline{\mathbf{Y}}=\sum_{j_{1}=1}^{J_{1}} \sum_{j_{2}=1}^{J_{2}} \sum_{j_{3}=1}^{J_{3}} g_{j_{1} j_{2} j_{3}} a_{j_{1}}^{(1)} \circ a_{j_{2}}^{(2)} \circ a_{j_{3}}^{(3)}+\underline{\mathbf{E}}$

$=\underline{\mathbf{G}} \times{ }_{1} \mathbf{A}^{(1)} \times{ }_{2} \mathbf{A}^{(2)} \times{ }_{3} \mathbf{A}^{(3)}+\underline{\mathbf{E}}=$

$\underline{\mathbf{G}} \times\{\mathbf{A}\}+\underline{\mathbf{E}}=\underline{\hat{\mathbf{Y}}}+\underline{\mathbf{E}}$

Here tensor $\underline{\hat{\mathbf{y}}}$ is an estimation of tensor $\underline{\mathbf{Y}}$ and it depends on the $\left(J_{1}, J_{2}, J_{3}\right)$ values, which are the dimensions of the core tensor $\underline{\mathbf{G}}$ and tensor $\underline{\mathbf{E}}$ denotes the estimation error. Most algorithms for the Nonnegative Tucker Decomposition (NTD) model are based on Alternative Least Square (ALS) minimization of the squared Euclidean distance [12] used as a global cost function subject to nonnegativity constraints, that is:

$\mathbf{D}_{F}(\underline{\mathbf{Y}} \| \underline{\mathbf{G}},\{\mathbf{A}\})=\frac{1}{2}\|\underline{\mathbf{Y}}-\underline{\hat{\mathbf{Y}}}\|^{2}$

Here the objective is to find the optimal component matrices $\mathbf{A}^{(n)} \in \mathbf{R}^{I_{n} \times J_{n}}$ and the core tensor $\underline{\mathbf{G}} \in \mathbf{R}^{J_{1} \times J_{2} \times J_{3}}$.
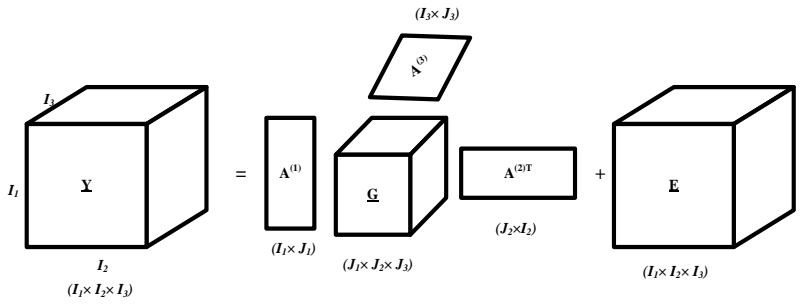

Fig. 3. Third-order Tucker decomposition.

Almost all the existing algorithms for Tucker decompositions [12] require certain processing based on the full tensor during the estimation. The realworld data often contain millions of elements. Full data processing (such as the inverse computation) is therefore impractical.We use the Hierarchical Nonnegative Tucker Decomposition algorithm in our proposed method. The reader is referred to [18] for more details of this algorithm. This will be shown in the proposed algorithm and presented in the next section.

\section{PROPOSED COMPRESSION ALGORITHM}

As described above, the TD algorithm can be reduced the spatial and spectral correlation simultaneously. In order to achieve higher compression ratios we propose a hybrid method based on DWT and TD ${ }^{1}[19]$.

The compression is performed using four steps. In the first step, 2DWT is applied to each spectral band of the HSIs in order to obtain 4 sub-images (approximate, diagonal, vertical and horizontal) for each spectral band (see Fig. 2). In the second step, the TD algorithm is applied to the four tensors. For each tensor, the size of the core tensor $\mathbf{G}$,i.e. $\left(J_{1}, J_{2}, J_{3}\right)$, was selected manually. The approximate tensor has the lowest frequency components containing most of the wavelet coefficient energy so the values of $\left(J_{1 A}, J_{2 A}, J_{3 A}\right)$ were set higher than those of other tensors. The values of $\left(J_{1 D}, J_{2 D}, J_{3 D}\right)$ for the diagonal tensor which contains the diagonal information were also set higher than the $\left(J_{1 H}, J_{2 H}, J_{3 H}\right)$ and $\left(J_{1 V}, J_{2 V}, J_{3 V}\right)$ values of horizontal and vertical tensors.

$\mathrm{CR}$ in this step can be considered as the ratio between the total number of bits in the original input data and the number of bits must be transmitted and is shown as:

$$
\begin{aligned}
C R & =\frac{\left(I_{1} \times I_{2} \times I_{3}\right)}{B} \\
B & =\frac{I_{1}}{2} \times J_{1 A}+\frac{I_{2}}{2} \times J_{2 A}+I_{3} \times J_{3 A}+J_{1 A} \times J_{2 A} \times J_{3 A} \\
& +\frac{I_{1}}{2} \times J_{1 D}+\frac{I_{2}}{2} \times J_{2 D}+I_{3} \times J_{3 D}+J_{1 D} \times J_{2 D} \times J_{3 D} \\
& +\frac{I_{1}}{2} \times J_{1 V}+\frac{I_{2}}{2} \times J_{2 V}+I_{3} \times J_{3 V}+J_{1 V} \times J_{2 V} \times J_{3 V} \\
& +\frac{I_{1}}{2} \times J_{1 H}+\frac{I_{2}}{2} \times J_{2 H}+I_{3} \times J_{3 H}+J_{1 H} \times J_{2 H} \times J_{3 H}
\end{aligned}
$$

In the third step, the 4 core tensors $\mathbf{G}$ and 12 matrices $\mathbf{A}^{(n)}$ should be transmitted.

Most elements of the core tensors $\underline{\mathbf{G}}$ (especially diagonal, vertical and horizontal core tensors) are nearly zero. The bitplane coding procedure is used to transmit the elements of these core tensors $\mathbf{G}$. The

\footnotetext{
1 The proposed algorithm is an improvement of our preliminary algorithms in [19] . Previous work lacks the use of adaptive arithmetic coding. We also added the impact of the proposed method on the linear unmixing and classification accuracy.
} 
bitplane coding is included two passes: the significance pass and the refinement. First, we define a significance map of a given threshold $\mathrm{T}$ and the element $g_{j_{1} j_{2} j_{3}}$. Suppose $\left|g_{j_{1} j_{2} j_{3}}\right|$ represents the absolute value of the core tensor element at the location $\left(j_{1}, j_{2}, j_{3}\right)$ and $s_{j_{1} j_{2} j_{3}}$ represents the significance state for the threshold $\mathrm{T}$ (where $\mathrm{T}$ is an integer power of 2),e.g.:

$s_{j_{1} j_{2} j_{3}}=\left\{\begin{array}{cc}1 & T \leq\left|g_{j_{1} j_{2} j_{3}}\right| \leq 2 T \\ 0 & O . W\end{array}\right.$

For $s_{j_{1} j_{2} j_{3}}=1$, the $g_{j_{1} j_{2} j_{3}}$ is considered as the significance element. The significance element must be encoded and removed from the core tensor; and the insignificant elements are preserved for the next bitplane. After that, the significance threshold is divided in half, and the process is repeated for the next pass. This process is repeated until the energy of encoded elements equal to or higher than $99.5 \%$ of that of the original core tensor. The selected elements and their positions must be transferred. There are many possible approaches for coding a significance map. Most wavelet-based coders use adaptive arithmetic coding (AAC) for lossless entropy coding [20]. We also use AAC for coding the significance element. Arithmetic coding is a variable-length lossless coding. Arithmetic coding does not require the transmission of codebook and so achieves a higher compression than Huffman coding. Arithmetic coding essentially amounts to computing the cumulative distribution function (CDF) of the probability of a sequence of symbols, and then representing the resulting numerical value in a binary code (see [21] for more details).

The columns of matrices $\mathbf{A}^{(n)}$ are called $a_{j_{n}}^{(n)}$ in the proposed algorithm, which are normalized vectors. The absolute value of $a_{j_{n}}^{(n)}$ elements are in the range $[0,1]$ and they are very close to each other. Therefore, in order to transfer the 12 matrices $\mathbf{A}^{(n)}$, a uniform quantization is used.

\section{Proposed Algorithm (DWT-TD)}

Input: Original HSIs $\underline{\mathbf{X}}$ (the size is $I_{1} \times I_{2} \times I_{3}$ )

1- Apply 2DWT to each spectral band to obtain 4 subimages (approximate, diagonal, vertical and horizontal tensors)

2- Apply TD algorithm to the four tensors individually.

Each tensor $\underline{\mathbf{Y}}$ has the size of $\frac{I_{1}}{2} \times \frac{I_{2}}{2} \times I_{3}$
$1 \leq J_{1} \leq \frac{I_{1}}{2}, 1 \leq J_{2} \leq \frac{I_{2}}{2}, 1 \leq J_{3} \leq \frac{I_{3}}{2}$

Initialize Nonnegative ALS for all $\mathbf{A}^{(n)}$ and $\underline{\mathbf{G}}$ Normalize all $a_{j_{n}}^{(n)}$ (for $n=1, \ldots, 3$ ) to unit length

$$
\begin{aligned}
& \quad \underline{\mathbf{E}}=\underline{\mathbf{Y}}-\underline{\hat{\mathbf{Y}}} \\
& \text { repeat } \\
& \quad \text { for } n=1 \text { to } 3 \text { do } \\
& \quad \text { for } j_{n}=1 \text { to } J_{n} \text { do } \\
& \mathbf{Y}_{(n)}^{\left(j_{n}\right)}=\mathbf{E}_{(n)}+a_{j_{n}}^{(n)}\left[\mathbf{G}_{(n)}\right]_{j_{n}} A^{\otimes_{-n} T} \\
& a_{j_{n}}^{(n)} \leftarrow\left[\mathbf { Y } _ { ( n ) } ^ { ( j _ { n } ) } \left[\underline{\left.\left.\left(\mathbf{G} \times{ }_{-n}\{\mathbf{A}\}\right)_{(n)}\right]_{j_{n}}^{T}\right]_{+}}\right.\right. \\
& a_{j_{n}}^{(n)} \leftarrow \frac{a_{j_{n}}^{(n)}}{\left\|a_{j_{n}}^{(n)}\right\|_{2}} \\
& \mathbf{E}_{(n)} \leftarrow \mathbf{Y}_{(n)}^{\left(j_{n}\right)}-a_{j_{n}}^{(n)}\left[\mathbf{G}_{(n)}\right]_{j_{n}} A^{\otimes_{-n} T} \\
& \quad \text { end } \\
& \quad \text { end } \\
& \quad \text { for each } j_{1}=1, \ldots, J_{1}, j_{2}=1, \ldots, J_{2}, j_{3}=1, \ldots, J_{3} \\
& \quad g_{j_{1} j_{2} j_{3}} \leftarrow g_{j_{1} j_{2} j_{3}}+\underline{\mathbf{E}} \overline{\times} a_{j_{1}}^{(1)} \overline{\times} a_{j_{2}}^{(2)} \overline{\times} a_{j 3}^{(3)} \\
& \quad \underline{\mathbf{E}} \leftarrow \underline{\mathbf{E}}+\Delta_{g_{j_{j} j_{2} j_{3}}} a_{j_{1}}^{(1)} \circ a_{j_{2}}^{(2)} \circ a_{j_{3}}^{(3)} \\
& \text { end } \\
& \text { until } a \text { stopping criterion is met }
\end{aligned}
$$

Output: 3 factors $\mathbf{A}^{(n)} \in \mathbf{R}^{I_{n} \times J_{n}}$ and a core tensor $\underline{\mathbf{G}} \in \mathbf{R}^{J_{1} \times J_{2} \times J_{3}}$

4-Quantize $\mathbf{A}^{(n)} \in \mathbf{R}^{I_{n} \times J_{n}}$ and encode core tensor $\underline{\mathbf{G}} \in \mathbf{R}^{J_{1} \times J_{2} \times J_{3}}$ Using AAC

In the fifth step, the transmitted data are decoded. Finally in the sixth step, the inverse of 2DWT is applied to reconstruct the images.

\section{Reconstructed Algorithm \\ 5- Decode the elements

$$
\underline{\hat{\mathbf{Y}}}=\underline{\hat{\mathbf{G}}} \times\{\hat{\mathbf{A}}\}
$$ \\ 6- Calculate the inverse 2DWT to reconstruct images \\ Output: Reconstructed images $\underline{\hat{\mathbf{x}}}$}

\section{COMPUTATIONAL COMPLEXITY}

In the following, we analyze the complexity of the algorithm.

We considered an $N$-tap filter bank and denoted $L_{b}$ as the number of wavelet decomposition levels in the spatial band. The complexity of applying 2DWT to the tensor $\underline{\mathbf{X}}$ with size of $I_{1} \times I_{2} \times I_{3}$ is 
$O\left(8 N I_{1} I_{2} I_{3}\left(1-2^{-2 L_{b}}\right) / 6\right)$ [22]. In the proposed algorithm we are using the (9/7) wavelet and also one level of decomposition, so the complexity of our algorithm is $O\left(9 I_{1} I_{2} I_{3} / 7\right)$

After applying 2DWT, each tensor (approximate, diagonal, vertical and horizontal tensors) has the size of $\frac{I_{1}}{2} \times \frac{I_{2}}{2} \times I_{3}$. The max complexity of the TD is of order $O\left(J_{A} I_{A}^{3}\right)$ [23], where $I_{A}$ is the average number of pixels of the approximate tensor and $J_{A}$ is the average of the dimensions of the approximate core tensor $\underline{\mathbf{G}}$.

$I_{A}=\frac{1}{3}\left(\frac{I_{1}}{2}+\frac{I_{2}}{2}+I_{3}\right)$

$J_{A}=\frac{1}{3}\left(J_{1 A}+J_{2 A}+J_{3 A}\right)$

Therefore, the total complexity of the proposed algorithm (DWT-TD) is of order

$4 \times O\left(J_{A} I_{A}{ }^{3}\right)+O\left(9 I_{1} I_{2} I_{3} / 7\right)$.

\section{EXPERIMENTAL RESULTS}

\section{A. Compression Results}

To measure the perceptual quality of images, the signal-to-noise ratio (SNR) can be well used. It actually estimates the quality of the reconstructed images $\underline{\hat{\mathbf{x}}}$ in comparison with the original ones $\underline{\mathbf{X}}$. The SNR in $\mathrm{dB}$ is defined as [24]:

$$
S N R_{d B}=10 \log _{10}\left(\frac{\|\underline{\mathbf{x}}\|^{2}}{\|\underline{\mathbf{x}}-\underline{\hat{\mathbf{x}}}\|^{2}}\right)
$$

where $\|\underline{\mathbf{X}}-\underline{\hat{\mathbf{x}}}\|^{2}=\sum_{k=1}^{I_{3}} \sum_{j=1}^{I_{2}} \sum_{i=1}^{I_{1}}\left(x_{i j k}-\hat{x}_{i j k}\right)^{2}$

For HSIs, one measures the bit rate as the number of bits per pixel per band (bpppb) which gives the average number of bits to represent a single sample of the hyperspectral data set.

Two popular AVIRIS datasets (Cuprite and Moffett) are used in our experiments ${ }^{1}$. These 16-bit radiance datasets have been cropped spatially to a size of $512 \times 512$ and composed of 224 spectral bands.

First, we apply the proposed algorithm (DWTTD) to these images. Next, we compare the SNRs achieved by the proposed algorithm with those obtained from the well-known techniques, i.e. 3DSPECK algorithm [5], combined PCA+JPEG2000 [6] on a set of HSIs. Table 3 shows the SNR versus different bpppb for the Cuprite and Moffett HSIs. In

\footnotetext{
${ }^{1}$ http://aviris.jpl.nasa.gov/html/aviris.freedata.html.
}

our experiments, DWT-TD has significantly improved SNR at high CRs (small bpppbs) especially when the $\mathrm{CR}$ is higher than 160 or bpppb is lower than 0.1 .

Table 3 SNR(dB) Values for, DWT-TD PCA+JPEG2000, 3D-SPECK

\begin{tabular}{|c|c|c|c|c|c|}
\hline \multirow{2}{*}{ Method } & \multicolumn{5}{|c|}{ bpppb } \\
\cline { 2 - 6 } & $\mathbf{0 . 0 5}$ & $\mathbf{0 . 1}$ & $\mathbf{0 . 2}$ & $\mathbf{0 . 5}$ & $\mathbf{1}$ \\
\hline \multicolumn{7}{|c|}{ Cuprite } \\
\hline DWT-TD & 49.5 & 52.1 & 52.6 & 54.2 & 58.1 \\
\hline PCA+JPEG2000 & 43.1 & 45.3 & 48.2 & 50.5 & 54.2 \\
\hline 3D SPECK & 34.7 & 37.1 & 40.8 & 46.6 & 50.1 \\
\hline \multicolumn{7}{|c|}{ Moffett Field } \\
\hline DWT-TD & 40.1 & 43.7 & 47.8 & 51.8 & 55.3 \\
\hline PCA+JPEG2000 & 34.7 & 39.3 & 43.6 & 47.2 & 51 \\
\hline 3D SPECK & 24.3 & 28.2 & 32.3 & 39.6 & 45.1 \\
\hline
\end{tabular}

\section{B. Classification Results}

Experiments are carried out using a hyperspectral data set which was taken over northwest Indiana's Indian Pine test site in June $1992^{2}$. This dataset include 145 by 145 pixels and 220 bands. First, 20 noisy bands due to water absorption have been removed. Second, from the 16 different land-cover classes available in the original ground truth, seven are discarded, since only few training samples are available for them .Third, $20 \%$ of the labeled samples for training and the rest for validation are considered. Pixel-based SVM classification are used with RBF kernel. The penalty parameter $C$ of SVM is varied in the range $c=\left\{10^{\circ}, \ldots, 10^{3}\right\}$ and the Gaussian kernel width is varied in the range $\sigma=\left\{10^{-1}, \ldots, 10^{3}\right\}$ using a fivefold cross validation. The best values obtained for these parameters are $c=200$ and $\sigma=0.5$. The one-against-one multiclass classification algorithm is used for the results presented in this paper. The SVM optimization problem is solved by using library LIBSVM [33]. As reported in Fig.4, the corresponding overall accuracy $(\mathrm{OA})$ is increased as the compression ratio increases. Because lossy compression can cause the local smoothing in the spatial and spectral domain then the noise effect is decreased. Therefore, the classification accuracy is increased. The similar results for some lossy compression methods were obtained in [10].

\section{Unmixing Results}

Vertex component analysis (VCA) is one of the popular methods for linear unmixing of HSIs [25]. We use this method for evaluating the impact of proposed method (DWT-TD) on the HSIs unmixing.

\footnotetext{
${ }^{2}$ http://dynamo.ecn.purdue.edu/ biehl/
} 
The Cuprite dataset with size of $250 \times 181$ pixels and 188 bands are considered in this section. The noisy bands as well as the water vapor absorption bands (including bands 1,2, 104-113, 148-167, and 221-224) are removed from the original 224-band data cube. This sub-image of Cuprite dataset was also used in [25] and the number of endmembers was considered as $p=14$. We also assume $\mathrm{p}=14$ and we apply DWT-TD to this dataset and we extract 14 endmembers from the original and compressed images (at different compression ratios) using VCA. Similar [10], we calculate the average spectral angle distance (SAD) between the endmembers obtained from the original images and the endmembers obtained from the compressed images. Fig.5. indicates that the proposed method (DWT+TD) has better performance (smaller SAD) in comparison with PCA+JPEG2000.

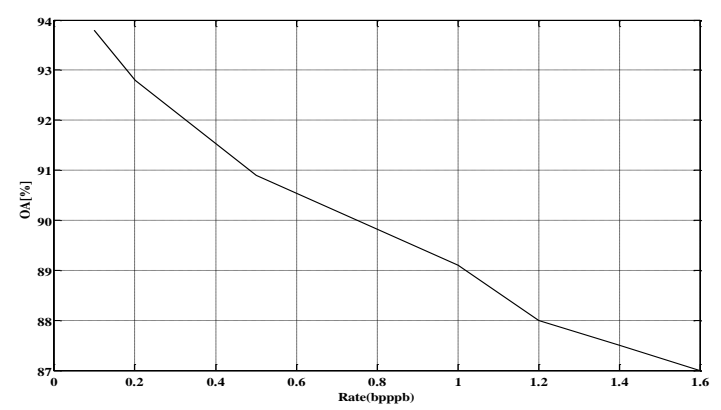

Fig.4 Classification accuracy of DWT-TD using SVM (RBF Kernel)

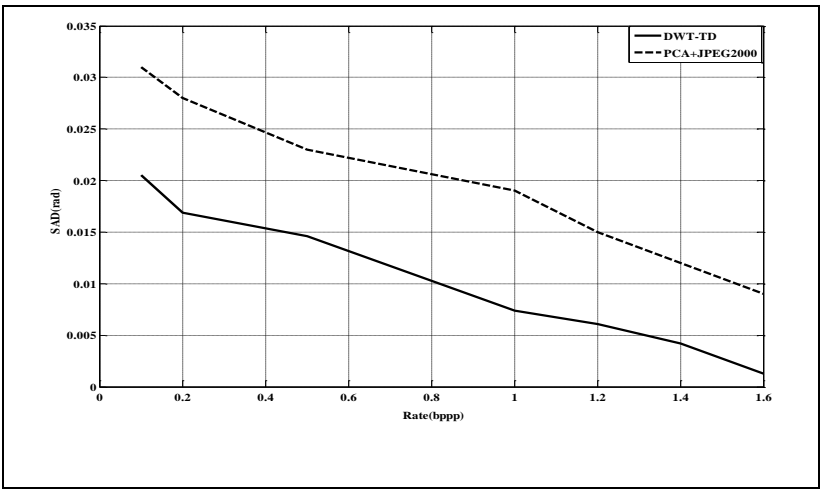

Fig.5 SAD(Rad) Versus bpppb for, DWT-TD and PCA+JPEG2000

\section{CONCLUSION}

In this paper, we introduced a new method for HSIs compression using DWT and TD. This is carried out by reducing the size of 3D tensors computed from four wavelet sub-images of the spectral bands of HSIs. The performance of the proposed algorithm on AVIRIS datasets yield the following results: 1) DWT-TD achieves smaller MSE in comparison with two state-of-the-art algorithms (3D-SPECK algorithm and combined PCA+JPEG2000) especially at bpppb lower than 0.12 2) the proposed algorithm achieves a better pixel-based SVM classification accuracy.

In future works, we aim to lower the computational load of the proposed method, for example by reducing the core tensor computations in order to speed up the compression process.

\section{ACKNOWLEDGMENT}

The authors would like to thank Prof. Landgrebe of Purdue University, West Lafayette, IN, for providing the Indian Pine AVIRIS data. The authors would also like to thank the reviewers for their valuable comments which have resulted in a number of improvements in the paper.

\section{REFERENCES}

[1] H. F. Grahn and P. Geladi, Techniques and applications of Hyperspectral image analysis: John Wiley \& Sons Ltd, Chichester, England, 2007.

[2] C. Huo, R. Zhang, and T. Peng, "Lossless Compression of Hyperspectral Images Based on Searching Optimal Multibands for Prediction," IEEE Geosci. and Remote Sens. Lett., vol. 6, pp. 339-343, Apr. 2009.

[3] J. Serra-Sagristà and F. Aulí-Llinàs, "Remote Sensing Data Compression," in Computational Intelligence for Remote Sensing. Berlin,Germany: Springer-Verlag,, pp. 27-61, Jun. 2008.

[4] J. Wang and C. Chang, "Independent component analysis-based dimensionality reduction with applications in Hyperspectral image analysis," IEEE Trans. Geosci. Remote Sens., vol. 44, pp. 1586-1600, Jun. 2006.

[5] X. Tang and W. A. Pearlman, "ThreeDimensional Wavelet-Based Compression of Hyperspectral Images," in Hyperspectral Data Compression, Chap.10, G. Motta, F. Rizzo, and A. Storer, Eds.: Springer, 2006, pp. 273-278.

[6] Q. Du and J. E. Fowler, "Hyperspectral image compression using JPEG2000 and principal component analysis," IEEE Geosci. and Remote Sens. Lett., vol. 4, pp. 201-205, Apr. 2007.

[7] G.Carvajal, B.Penna, and E.Magli, "Unified lossy and near-lossless hyperspectral image compression based on JPEG 2000," IEEE Geosci. and Remote Sens. Lett., vol. 5, pp. 593-597, Oct. 2008.

[8] J. Zhang, J. E. Fowler, and G.Liu, "Lossy-toLossless compression of hyperspectral imagery using three-Dimensional TCE and an integer KLT," IEEE Geosci. and Remote Sens. Lett., vol. 5, pp. 814-818, Oct. 2008. 
[9] H.Chen, Y.Zhang, J.Zhang, and Y.Chen, "A BOI-Preserving-Based compression method for hyperspectral images," IEEE Trans. Geosci. Remote Sens. , vol. 48, pp. 3913-3923, Nov. 2010.

[10] F.Garcia-Vilchez, J.Munoz-Mari, I. B. M.Zortea, V.Gonzalez-Ruiz, G.Camps-Valls, A.Plaza, and J.Serra-Sagrista, "On the Impact of Lossy Compression on Hyperspectral Image Classification and Unmixing," IEEE Geosci. and Remote Sens. Lett., vol. 8, pp. 253-257, Mar. 2011.

[11] N. Renard and S. Bourennane, "Dimensionality reduction based on tensor modeling for classification methods," IEEE Trans. Geosci. Remote Sens., vol. 47, pp. 1123-1131, Apr. 2009.

[12] T. Kolda and B. Bader, "Tensor decompositions and applications," SIAM Review, vol. 51, pp. 455-500, Sep. 2009.

[13] S. Mallat, A Wavelet Tour of Signal Porocessing: Academic,San Diego 1998.

[14] R.C.Gonzalez and R.E.woods, Digital Image Processing: Prentice Hall, 2002.

[15] W.Kim and C-C.Li, "A Study on Preconditioning Multiwavelet Systems for Image Compression " in Wavelet analysis and its applications, Y. Y. Tang, P. C.Yuen, and C-h.Li, Eds., 2001, pp. 22-36

[16] A. Cohen, I. Daubechies, and J.-C. Feauveau, "Biorthogonal bases of compactly supported wavelets," Communications on Pure and Applied Mathematics, vol. 45, pp. 485-560, May 1992.

[17] M. Antonini, M. Barlaud, P. Mathieu, and I. Daubechies, "Image coding using wavelet transform," IEEE Transactions on Image Processing, vol. 1, pp. 205-220, April 1992.

[18] A. Cichocki, R. Zdunek, A. H. Phan, and S. Amari, Nonnegative matrix and tensor factorizations: Wiley, Chichester, 2009.

[19] A.Karami, M.Yazdi, and G.Mercier, "Hyperspectral image compression based on tucker decomposition and wavelet transform," in 3rd Workshop on Hyperspectral Image and Signal Processing: Evolution in Remote Sensing (WHISPERS), Lisbon, 6-9 June 2011 pp. 1 - 4

[20] I. H. Witten, R. M. Neal, and J. G. Cleary, "Arithmetic coding for data compression " Communications of the ACM, vol. 30, pp. 520-540, 1987.

[21] K.S.Thyagarajan: John Wiley \& Sons, Inc., 2011.

[22] B. Penna, T. Tillo, E. Magli, and G. Olmo, "Progressive 3-D Coding of Hyperspectral Images Based on JPEG 2000," IEEE Geosci. and Remote Sens. Lett., vol. 3, pp. 125-129, Jan. 2006.
[23] L. D. Lathauwer, B. D. Moor, and J. Vandewalle, "A multilinear singular value decomposition," SIAM Journal Matrix Analysis Application, vol. 21, pp. 1253-1278, Apr. 2000.

[24] K. Jain, Fundamentals of Digital Image Processing: Printice-Hall, 1989.

[25] J. M. P. Nascimento and J. M. Bioucas-Dias, "Vertex component analysis : A fast algorithm to unmix hyperspectral data," IEEE Trans. Geosci. Remote Sens, vol. 43, pp. 898-910, Apr. 2005.

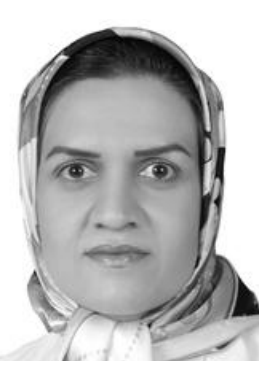

Azam Karami received the B.Sc. degree in Electronic Engineering from Shahid Beheshti University, Tehran, Iran, in 2003 and the M.Sc. degree in Control Engineering from Shahid Bahonar University, Kerman, Iran, in 2006. She is currently working toward the Ph.D. degree in Communication System at the Department of Electrical Engineering, Shiraz University, Shiraz, Iran. Her primary research interests include image and video compression and denoising, remote sensing, multidimentional signal processing, and optimization techniques.

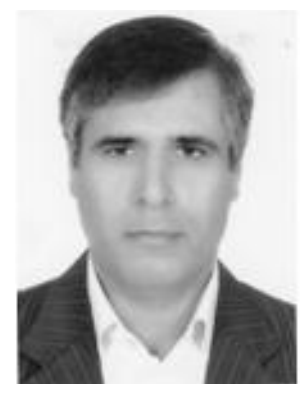

Mehran Yazdi received the B.Sc. degree in communication systems from the Department of Electrical Engineering, Shiraz University, Shiraz, Iran, in 1992 and the M.Sc. and Ph.D. degrees in digital vision and image processing from the Department of Electrical Engineering, Laval University, Quebec, QC, Canada, in 1996 and 2003, respectively. He is currently an Associate Professor in the Department of Communications and Electronics Engineering, Shiraz University. He conducted several projects in the area of hyperspectral image compression and denoising, CT metal artifact reduction, and video compression. His major research interests are in the field of image/video processing, remote sensing, multidimentional signal processing, and medical image analysis. 


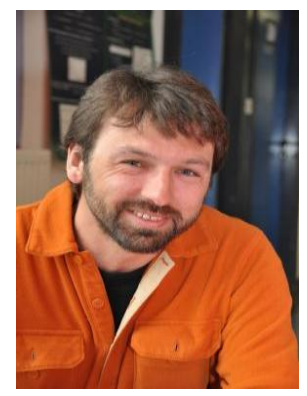

Grégoire Mercier was born in France in 1971. He received the Engineer degree from the Institute National des Télécommunications, Evry, France, in 1993 and the Ph.D. degree and the Habilitation à Diriger des Recherches from the University of Rennes I, Rennes, France, in 1999 and 2007, respectively. Since 1999, he has been with Télécom Bretagne, where since early 2010 he has been a Professor in the Image and Information Processing Department (ITI). He was a Visiting Researcher at DIBE (University of Genoa, Italy) from March to May 2006, where he developed change detection technique for heterogeneous data. $\mathrm{He}$ was also a visiting researcher at CNES (France) from April to June 2007 to take part of the Orfeo Toolbox development. His research interests are in remote sensing image compression and segmentation, especially in hyperspectral and synthetic aperture radar. Actually, his research is dedicated to change detection and combating pollution.

Prof. Mercier is President of the French Chapter of IEEE Geoscience and Remote Sensing Society. He is an Associate Editor for the IEEE GEOSCIENCE AND REMOTE SENSING LETTERS. 